\title{
Effective spin-wave action for ordered Heisenberg antiferromagnets in a magnetic field
}

\author{
Nils Hasselmann, Florian Schütz, Ivan Spremo, and Peter Kopietz \\ Institut für Theoretische Physik, Universität Frankfurt, \\ Max-von-Laue-Strasse 1, 60438 Frankfurt, Germany
}

(Dated: November 29, 2005)

\begin{abstract}
We derive the effective long-wavelength Euclidean action for the antiferromagnetic spin-waves of ordered quantum antiferromagnets subject to a uniform magnetic field. We point out that the magnetic field dependence of the spin-wave dispersion predicted by the usual $O(3)$-quantum nonlinear sigma model disagrees with spin-wave theory. We argue that the nonlinear sigma model does not take into account all relevant spin-wave interactions and derive a modified effective action for the long-wavelength spin-waves which contains an additional quartic interaction. At zero temperature the corresponding vertex is relevant in the renormalization group sense below three dimensions.

PACS numbers: 75.10.Jm, 75.30.Ds, 75.50.Cx
\end{abstract}

\section{INTRODUCTION}

Recently, two of us derived a new effective field theory $\underline{\underline{1}}$ for transverse spin fluctuations in ordered quantum antiferromagnets. Our approach is based on the HolsteinPrimakoff transformation, ${ }^{2}$ which maps the original spin Hamiltonian onto a bosonic many-body Hamiltonian. However, rather than working directly with the canonical Holstein-Primakoff bosons, we express them in terms of Hermitian field operators representing staggered and uniform transverse spin fluctuations. $\underline{\underline{3}}$ The main advantages of this procedure are: (a) the suppression of the effective interaction between antiferromagnetic magnons at long wavelengths is manifest; (b) the relation between Holstein-Primakoff bosons and the fields of the nonlinear sigma mode $\underline{14}^{4}$ (NLSM) representing the transverse staggered spin fluctuation is made precise; (c) the zero wave vector modes of the uniform and staggered magnetization can be easily treated within this approach, $\frac{3}{\underline{3}}$ which is important in the study of finite magnets; (d) the transverse ferromagnetic fluctuations may be eliminated to yield an effective theory involving only transverse antiferromagnetic fluctuations. This is most easily done within a path integral approach; the resulting effective action coincides with the NLSM at the Gaussian level. Although the interaction terms of the effective action differ from those of the NLSM, the renormalization group flows of both theories agree to one-loop order $\frac{1}{n}$

Here, we extend the Hermitian operator approach to antiferromagnets in a uniform magnetic field. In this case, the differences between this approach and the NLSM become more substantial. Already at the Gaussian level the two theories differ: while the Hermitian operator approach reproduces by construction the correct spin-wave dispersion known from spin-wave theory, the NLSM does not. More severely, we show below that a quartic interaction term present in the Hermitian operator approach is absent in the NLSM.

We shall constrain the analysis here to a Heisenberg model on a hypercubic lattice with nearest neighbor antiferromagnetic coupling $J>0$. It is however straightforward to extend the results to general bipartite lat- tices. This would also cover a recently synthesized metalorganic $S=5 / 2$ quantum antiferromagnet on a distorted honeycomb lattice,,$\frac{5}{-}$ which is bipartite. In presence of a uniform external field $\boldsymbol{h}$ (measured in units of energy), the Hamiltonian is given by

$$
\hat{H}=J \sum_{\langle i j\rangle} \boldsymbol{S}_{i} \cdot \boldsymbol{S}_{j}-\sum_{i} \boldsymbol{h} \cdot \boldsymbol{S}_{i}
$$

Here, $\boldsymbol{S}_{i}$ are spin operators normalized such that $\boldsymbol{S}_{i}^{2}=$ $S(S+1)$, and the first sum is over nearest neighbor pairs $\langle i j\rangle$ of a hypercubic lattice with lattice constant $a$.

Our main interest is a description of the low-energy and long-wavelength properties of the Heisenberg model (11). A standard approach to this problem is the $O(3)$ quantum NLSM whose effective action describes only the relevant staggered spin fluctuations. According to Refs. [6.7], a magnetic field can be taken into account within the NLSM approach by replacing the derivative $\partial_{\tau}$ with respect to the imaginary time $\tau$ with a covariant derivative (or Lie derivative, see Ref. [8])

$$
\partial_{\tau} \rightarrow \partial_{\tau}-i \boldsymbol{h} \times .
$$

With this minimal coupling the NLSM in a uniform magnetic field has the form

$$
\begin{array}{r}
S_{\mathrm{NLSM}}[\boldsymbol{\Omega}]=\frac{\rho_{0}}{2} \int_{0}^{\beta} d \tau \int d^{D} r\left[\sum_{\mu=1}^{D}\left(\partial_{\mu} \boldsymbol{\Omega}\right)^{2}\right. \\
\left.+\frac{1}{c_{0}^{2}}\left(\partial_{\tau} \boldsymbol{\Omega}-i \boldsymbol{h} \times \boldsymbol{\Omega}\right)^{2}\right],
\end{array}
$$

where the unit vector $\boldsymbol{\Omega}(\tau, \boldsymbol{r})$ represents the slowly fluctuating staggered magnetization, $\rho_{0}$ and $c_{0}$ are the spin stiffness and spin-wave velocity at temperature $T=$ $1 / \beta=0$, and $\partial_{\mu}=\partial / \partial r_{\mu}$ is the spatial derivative in direction $\mu=1, \ldots, D$. Numerical values of $\rho_{0}$ and $c_{0}$ must be computed microscopically, e. g. by means of a $1 / S$-expansion ${ }^{\underline{4}}$ To leading order in $1 / S$, one has $\rho_{0} \approx J S^{2} a^{2-D}$ and $c_{0} \approx 2 D^{1 / 2} J S a$. For later reference, we note that the classical uniform transverse susceptibility is $\chi_{0}=\rho_{0} / c_{0}^{2}$ with $\chi_{0} \approx\left(4 D J a^{D}\right)^{-1}$ to leading order in $1 / S$. The classical uniform magnetization per volume is $\boldsymbol{M}_{0}=\chi_{0} \boldsymbol{h}$. 


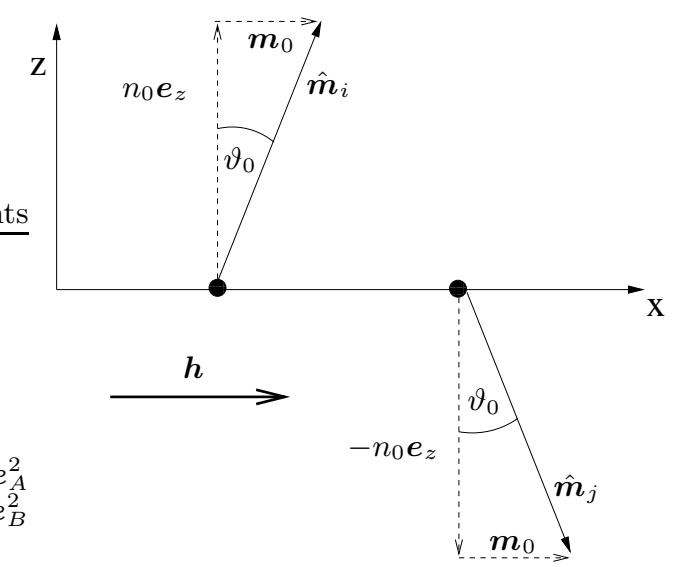

FIG. 1: Spin configuration $\left\langle\boldsymbol{S}_{i}\right\rangle=\boldsymbol{S \boldsymbol { m } _ { i }}$ in the classical ground state of a two-sublattice antiferromagnet subject to a uniform magnetic field $\boldsymbol{h}=h \boldsymbol{e}_{x}$, where $\boldsymbol{r}_{i}$ belongs to sublattice $A$ and $\boldsymbol{r}_{j}$ to sublattice $B$. Here $\boldsymbol{m}_{0}=\boldsymbol{h} /(4 D J S)$ and $n_{0}=$ $\sqrt{1-m_{0}^{2}}$.

\section{SPIN-WAVE DISPERSION}

In absence of a magnetic field, the NLSM predicts a doubly degenerate antiferromagnetic spin-wave mode with long-wavelength dispersion $E_{\boldsymbol{k}}=c_{0}|\boldsymbol{k}|$, in agreement with linear spin-wave theory $\underline{4}$ Rather surprisingly, however, in presence of a magnetic field the longwavelength dispersion derived from the NLSM in Eq. (3) disagrees with spin-wave theory. To see this, let us recall the spin-wave dispersion predicted by linear spinwave theory. In this approach the spin operators are expanded in deviations from the classical ground state spin configuration, using either the Holstein-Primakoff2 ${ }^{2}$ or the Dyson-Maleev ${ }^{9}$ transformation. The classical ground state of a quantum antiferromagnet in a uniform external field is canted, where the staggered magnetization is perpendicular to the magnetic field, as shown in Fig. 1] To leading order in $1 / S$, spin-wave theory ${ }^{5.10 .11}$ predicts two transverse spin-wave modes, one gapless and one gapped, with long-wavelength dispersions (see Eqs. (1720) below)

$$
E_{\boldsymbol{k}+} \approx\left[h^{2}+c_{+}^{2} \boldsymbol{k}^{2}\right]^{1 / 2}, \quad E_{\boldsymbol{k}-} \approx c_{-}|\boldsymbol{k}|,
$$

where the spin-wave velocities are

$$
c_{+}=c_{0} \sqrt{1-3 m_{0}^{2}}, c_{-}=c_{0} \sqrt{1-m_{0}^{2}} .
$$

Here, $m_{0}=\left|\boldsymbol{m}_{0}\right|$ is the length of the normalized classical uniform magnetization

$$
\boldsymbol{m}_{0}=a^{D} \frac{\boldsymbol{M}_{0}}{S}=\frac{\boldsymbol{h}}{4 D J S},
$$

which is related to the classical canting angle $\vartheta_{0}$ between the direction of the staggered magnetization and the local magnetic moments by $m_{0}=\sin \vartheta_{0}$. In general, the classical canting angle is renormalized by quantum fluctuations. 5.10

Let us compare Eqs. (45) with the dispersion relation obtained from the NLSM. Denoting by $\boldsymbol{e}_{\alpha}$ the unit vectors in the fixed directions $\alpha=x, y, z$ and assuming $\boldsymbol{h}=h \boldsymbol{e}_{x}$ to point along the $x$-axis, we write

$$
\boldsymbol{\Omega}=\Pi_{\|} \boldsymbol{e}_{x}+\Pi_{\perp} \boldsymbol{e}_{y}+\left[1-\Pi_{\|}^{2}-\Pi_{\perp}^{2}\right]^{1 / 2} \boldsymbol{e}_{z},
$$

and expand the action $S_{\mathrm{NLSM}}[\boldsymbol{\Omega}]$ to quadratic order in the fluctuations $\Pi_{\|}$and $\Pi_{\perp}$ transverse to the direction of the staggered magnetization. One easily finds from Eq. (3) that the spin-wave mode $\Pi_{\|}$polarized parallel to the magnetic field is gapped with energy dispersion

$$
E_{\boldsymbol{k} \|}=\left[h^{2}+c_{0}^{2} \boldsymbol{k}^{2}\right]^{1 / 2},
$$

while the spin-wave mode $\Pi_{\perp}$ polarized perpendicular to the magnetic field has a gapless linear dispersion,

$$
E_{\boldsymbol{k} \perp}=c_{0}|\boldsymbol{k}| \text {. }
$$

The spin-wave spectrum of the NLSM given in Eqs. (8) and (9) does not reproduce the correct magnetic fielddependence of the spin-wave spectrum in Eqs. (45) obtained via conventional spin-wave theory. Obviously, the above minimal coupling (2) does not account for order $(h / J)^{2}$ corrections to the dispersion, even though the qualitative aspects of the dispersion are correctly described.

\section{EFFECTIVE ACTION}

In Ref. [1] we have developed a new method to derive the effective action for staggered transverse spinfluctuations. We here sketch the main steps of the derivation adapted to a quantum antiferromagnet subject to a uniform magnetic field ${ }^{12}$ Starting point is the representation of spin operators in terms of canonical boson operators $b_{i}$ and $b_{i}^{\dagger}$ using the Holstein-Primakoff transformation ${ }^{2}$. Let us denote by $\hat{\boldsymbol{m}}_{i}=\left\langle\boldsymbol{S}_{i}\right\rangle / S$ the directions of the spins in the true ground state and introduce a local right-handed orthogonal triad of unit vectors $\boldsymbol{e}_{i}^{(1)}, \boldsymbol{e}_{i}^{(2)}, \hat{\boldsymbol{m}}_{i}$. As explained in Ref. [13] there is a $U(1)$ gauge freedom in the choice of the transverse vectors $\boldsymbol{e}_{i}^{(1)}$ and $\boldsymbol{e}_{i}^{(2)}$. Defining the spherical basis vectors $\boldsymbol{e}_{i}^{p}$ $=\boldsymbol{e}_{i}^{(1)}+i p \boldsymbol{e}_{i}^{(2)}, p= \pm$, we express the components of the spin operator $\boldsymbol{S}_{i}$ in terms of canonical boson operators $b_{i}$ and $b_{i}^{\dagger}$ as follows, ${ }^{2}$

$$
\boldsymbol{S}_{i}=S_{i}^{\|} \hat{\boldsymbol{m}}_{i}+\boldsymbol{S}_{i}^{\perp}=S_{i}^{\|} \hat{\boldsymbol{m}}_{i}+\frac{1}{2} \sum_{p= \pm} S_{i}^{-p} \boldsymbol{e}_{i}^{p},
$$

with

$$
\begin{aligned}
S_{i}^{\|} & =S-n_{i}, \quad n_{i}=b_{i}^{\dagger} b_{i}, \\
S_{i}^{+} & =(2 S)^{1 / 2}\left(1-\frac{n_{i}}{2 S}\right)^{1 / 2} b_{i}
\end{aligned}
$$


and $S_{i}^{-}=\left(S_{i}^{+}\right)^{\dagger}$. The Heisenberg model (11) can then be written as a bosonic many-body Hamiltonian. ${ }^{\frac{5}{r}}$ We expand around the classical ground state configuration (see Fig. (1)

$$
\hat{\boldsymbol{m}}_{i}=\zeta_{i} n_{0} \boldsymbol{e}_{z}+m_{0} \boldsymbol{e}_{x}
$$

and choose

$$
\boldsymbol{e}_{i}^{(1)}=\boldsymbol{e}_{y}, \quad \boldsymbol{e}_{i}^{(2)}=-\zeta_{i} n_{0} \boldsymbol{e}_{x}+m_{0} \boldsymbol{e}_{z},
$$

where $\zeta_{i}=1\left(\zeta_{i}=-1\right)$ for $\boldsymbol{r}_{i} \in A\left(\boldsymbol{r}_{i} \in B\right)$. Here, $n_{0}=\sqrt{1-m_{0}^{2}}=\cos \vartheta_{0}$. To diagonalize the quadratic part of this Hamiltonian, we first perform a Fourier transformation in the sublattice basis

$$
b_{i}= \begin{cases}(2 / N)^{1 / 2} \sum_{\boldsymbol{k}} e^{i \boldsymbol{k} \cdot \boldsymbol{r}_{i}} A_{\boldsymbol{k}}, & \boldsymbol{r}_{i} \in A, \\ (2 / N)^{1 / 2} \sum_{\boldsymbol{k}} e^{i \boldsymbol{k} \cdot \boldsymbol{r}_{i}} B_{\boldsymbol{k}}, & \boldsymbol{r}_{i} \in B,\end{cases}
$$

where the momentum sums are over the reduced (antiferromagnetic) Brillouin zone. After introducing the symmetric and antisymmetric combinations $C_{\boldsymbol{k} \sigma}=$ $2^{-1 / 2}\left[A_{\boldsymbol{k}}+\sigma B_{\boldsymbol{k}}\right], \sigma= \pm$, we apply the Bogoliubov transformation

$$
\left(\begin{array}{c}
C_{\boldsymbol{k} \sigma} \\
C_{-\boldsymbol{k} \sigma}^{\dagger}
\end{array}\right)=\left(\begin{array}{cc}
u_{\boldsymbol{k} \sigma} & -\sigma v_{\boldsymbol{k} \sigma} \\
-\sigma v_{\boldsymbol{k} \sigma} & u_{\boldsymbol{k} \sigma}
\end{array}\right)\left(\begin{array}{c}
\hat{\Psi}_{\boldsymbol{k} \sigma} \\
\hat{\Psi}_{-\boldsymbol{k} \sigma}^{\dagger}
\end{array}\right)
$$

where

$$
\begin{aligned}
& u_{\boldsymbol{k} \sigma}=\left[\frac{1+\sigma m_{0}^{2} \gamma_{\boldsymbol{k}}+\epsilon_{\boldsymbol{k} \sigma}}{2 \epsilon_{\boldsymbol{k} \sigma}}\right]^{1 / 2}, \\
& v_{\boldsymbol{k} \sigma}=\left[\frac{1+\sigma m_{0}^{2} \gamma_{\boldsymbol{k}}-\epsilon_{\boldsymbol{k} \sigma}}{2 \epsilon_{\boldsymbol{k} \sigma}}\right]^{1 / 2},
\end{aligned}
$$

with

$$
\epsilon_{\boldsymbol{k} \sigma}=\left[\left(1+\sigma m_{0}^{2} \gamma_{\boldsymbol{k}}\right)^{2}-\left(n_{0}^{2} \gamma_{\boldsymbol{k}}\right)^{2}\right]^{1 / 2} .
$$

Here, $\gamma_{\boldsymbol{k}}=D^{-1} \sum_{\mu} \cos \left(\boldsymbol{k} \cdot \boldsymbol{a}_{\mu}\right)$, where $\boldsymbol{a}_{\mu}, \mu=1 \ldots D$, are the $D$ primitive lattice vectors of the hypercubic lattice. The quadratic part of the effective boson Hamiltonian assumes then the form of non-interacting harmonic oscillators,$\frac{5,12}{,}$

$$
\hat{H}_{2}=\sum_{\boldsymbol{k}, \sigma} E_{\boldsymbol{k} \sigma}\left[\hat{\Psi}_{\boldsymbol{k} \sigma}^{\dagger} \hat{\Psi}_{\boldsymbol{k} \sigma}+\frac{1}{2}\right]
$$

where the operators $\hat{\Psi}_{\boldsymbol{k} \sigma}$ satisfy the canonical bosonic commutation relations,

$$
\left[\hat{\Psi}_{\boldsymbol{k} \sigma}, \hat{\Psi}_{\boldsymbol{k}^{\prime} \sigma^{\prime}}^{\dagger}\right]=\delta_{\boldsymbol{k}, \boldsymbol{k}^{\prime}} \delta_{\sigma, \sigma^{\prime}},
$$

and the energy dispersion of the two spin-wave modes are given by

$$
E_{\boldsymbol{k} \sigma}=2 D J S \epsilon_{\boldsymbol{k} \sigma}
$$

In the limit of long wavelengths Eq. (20) reduces to the energy dispersions given in Eqs. (45).
To derive the long-wavelength effective action for the staggered transverse spin fluctuations, we need the precise relation between the Bogoliubov quasi-particle operators $\hat{\Psi}_{k \sigma}$ and the field operators $\hat{\Pi}_{k \sigma}$ representing the transverse fluctuations of the staggered magnetization. In a Euclidean path integral approach, these operators correspond to continuum fields $\Pi_{\boldsymbol{k} \sigma}(\tau)$ which are analogs of the fields $\Pi_{\boldsymbol{k} \|}(\tau)$ and $\Pi_{\boldsymbol{k} \perp}(\tau)$ of the NLSM. The relation between these two different parameterizations of the spin fluctuations is simply $\underline{1}^{\underline{1}}$

$$
\begin{aligned}
& \hat{\Psi}_{\boldsymbol{k}+}=-i\left(\chi_{0} / 2 V E_{\boldsymbol{k}+}\right)^{1 / 2}\left[E_{\boldsymbol{k}+} \hat{\Pi}_{\boldsymbol{k}+}+i \chi_{0}^{-1} \hat{\Phi}_{\boldsymbol{k}+}\right], \\
& \hat{\Psi}_{\boldsymbol{k}-}=\left(\chi_{0} / 2 V E_{\boldsymbol{k}-}\right)^{1 / 2}\left[E_{\boldsymbol{k}-} \hat{\Pi}_{\boldsymbol{k}-}+i \chi_{0}^{-1} \hat{\Phi}_{\boldsymbol{k}-}\right]
\end{aligned}
$$

where $V=N a^{D}$ is the volume of the system. One easily verifies that the pairs $\hat{\Pi}_{\sigma}, \hat{\Phi}_{\sigma}$ satisfy commutation relations of canonically conjugate bosonic field operators,

$$
\left[\hat{\Pi}_{\boldsymbol{k} \sigma}, \hat{\Phi}_{\boldsymbol{k}^{\prime} \sigma^{\prime}}\right]=i V \delta_{\boldsymbol{k},-\boldsymbol{k}^{\prime}} \delta_{\sigma, \sigma^{\prime}} .
$$

The field operators $\hat{\Pi}_{\boldsymbol{k} \sigma}$ and $\hat{\Phi}_{\boldsymbol{k} \sigma}$ have a simple interpretation in terms of transverse staggered and uniform spin components. The Fourier transformed spin operators on the $A$ and $B$ sublattice are given by

$$
\boldsymbol{S}_{A / B, \boldsymbol{k}}=(2 / N)^{1 / 2} \sum_{\boldsymbol{r}_{i} \in A / B} e^{-i \boldsymbol{k} \cdot \boldsymbol{r}_{i}} \boldsymbol{S}_{i} .
$$

Using Eqs. (1013) we find that, to leading order in $1 / S$ and $m_{0}$, the transverse staggered components of the spins are given by 14

$$
\begin{aligned}
& S_{\mathrm{st}, \boldsymbol{k}}^{(1)}=\frac{1}{\sqrt{2}}\left(S_{A, \boldsymbol{k}}^{(1)}-S_{B, \boldsymbol{k}}^{(1)}\right) \approx\left(S / a^{D}\right) N^{-1 / 2} \hat{\Pi}_{\boldsymbol{k}-}, \\
& S_{\mathrm{st}, \boldsymbol{k}}^{(2)}=\frac{1}{\sqrt{2}}\left(S_{A, \boldsymbol{k}}^{(2)}+S_{B, \boldsymbol{k}}^{(2)}\right) \approx-\left(S / a^{D}\right) N^{-1 / 2} \hat{\Pi}_{\boldsymbol{k}+}
\end{aligned}
$$

In contrast, the transverse components of the uniform magnetization are related to the operators $\hat{\Phi}_{\boldsymbol{k} \sigma}$,

$$
\begin{aligned}
& S_{\boldsymbol{k}}^{(1)}=\frac{1}{\sqrt{2}}\left(S_{A, \boldsymbol{k}}^{(1)}+S_{B, \boldsymbol{k}}^{(1)}\right) \approx N^{-1 / 2} \hat{\Phi}_{\boldsymbol{k}+}, \\
& S_{\boldsymbol{k}}^{(2)}=\frac{1}{\sqrt{2}}\left(S_{A, \boldsymbol{k}}^{(2)}-S_{B, \boldsymbol{k}}^{(2)}\right) \approx N^{-1 / 2} \hat{\Phi}_{\boldsymbol{k}-} .
\end{aligned}
$$

In terms of these operators, our quadratic spin-wave Hamiltonian (18) can be written as

$$
\hat{H}_{2}=\frac{1}{2 V} \sum_{\boldsymbol{k}, \sigma}\left[\chi_{0}^{-1} \hat{\Phi}_{-\boldsymbol{k} \sigma} \hat{\Phi}_{\boldsymbol{k} \sigma}+\chi_{0} E_{\boldsymbol{k} \sigma}^{2} \hat{\Pi}_{-\boldsymbol{k} \sigma} \hat{\Pi}_{\boldsymbol{k} \sigma}\right] .
$$

The effective Euclidean action $S_{\text {eff }}\left[\Pi_{\sigma}\right]$ for the staggered spin fluctuations can now be obtained by writing the partition function as a phase space path integral over the fields $\Pi_{\boldsymbol{k} \sigma}(\tau)$ and $\Phi_{\boldsymbol{k} \sigma}(\tau)$ associated with the above operators and subsequently integrating over the $\Phi_{\sigma}$-fields which represent gapped ferromagnetic fluctuations. At 
the level of a Gaussian approximation, we obtain in this way $S_{\text {eff }} \approx S_{\text {eff }}^{(2)}$ with

$$
S_{\mathrm{eff}}^{(2)}\left[\Pi_{\sigma}\right]=\frac{\chi_{0}}{2 \beta V} \sum_{K, \sigma}\left(\omega_{n}^{2}+E_{\boldsymbol{k} \sigma}^{2}\right) \Pi_{-K \sigma} \Pi_{K \sigma} .
$$

Here, we have combined momenta $\boldsymbol{k}$ and bosonic Matsubara frequencies $\omega_{n}$ in a composite label $K=\left(\boldsymbol{k}, i \omega_{n}\right)$. We have further defined

$$
\Pi_{K \sigma}=\int_{0}^{\beta} d \tau e^{i \omega_{n} \tau} \Pi_{\boldsymbol{k} \sigma}(\tau) .
$$

At long wavelengths the effective action (27) has the same form as the corresponding Gaussian part of the action of the NLSM. However, in contrast to the NLSM (3), our effective action (27) has the correct spin-wave dispersion, even for short wavelengths.

We have calculated the leading corrections to the effective action $S_{\text {eff }}\left[\Pi_{\sigma}\right]$ for staggered spin fluctuations arising from spin-wave interactions in the Holstein-Primakoff approach ${ }^{12}$ Keeping only interaction terms which become relevant below three dimensions and thus omitting terms which are marginal in $D=1$, we find in the continuum limit

$$
\begin{aligned}
S_{\mathrm{eff}}\left[\Pi_{\sigma}\right] & =\frac{\chi_{0}}{2 \beta V} \sum_{K, \sigma}\left(\omega_{n}^{2}+c_{\sigma}^{2} \boldsymbol{k}^{2}+r_{\sigma}\right) \Pi_{-K \sigma} \Pi_{K \sigma} \\
& -i \chi_{0} h \int_{0}^{\beta} d \tau \int d^{D} r \Pi_{+}^{2} \partial_{\tau} \Pi_{-} \\
& -\frac{\chi_{0} h^{2}}{8} \int_{0}^{\beta} d \tau \int d^{D} r \Pi_{+}^{2}\left(\Pi_{+}^{2}+\Pi_{-}^{2}\right) .
\end{aligned}
$$

Here, the $c_{\sigma}$ are the Holstein-Primakoff results for the spin-wave velocities, which for large $S$ are given in Eq. (5). To leading orders in $1 / S$, the values of the gap parameters are $r_{+}=h^{2}$ and $r_{-}=0$. For symmetry reasons, the true spin-wave spectrum of the $\Pi_{-}$-mode must remain gapless at vanishing wave-vector (Goldstone

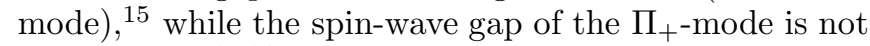
renormalized ${ }^{15.16}$. In a renormalization group analysis, this requires fine tuning such that at the fixed point these conditions are met. While the cubic term in Eq. (29) corresponds precisely to the cubic (Berry phase) term in the NLSM (31), the quartic interaction between the field components is absent if the magnetic field is included in the NLSM by the minimal coupling (2).

\section{SUMMARY AND CONCLUSION}

In this work we have used the Holstein-Primakoff transformation to derive an effective action for staggered transverse spin fluctuations of quantum Heisenberg antiferromagnets in uniform magnetic fields. Our effective action contains an additional quartic interaction between the field components which is not contained in the NLSM. It is easy to see that at zero temperature the quartic interaction vertex in Eq. (29) is relevant in the renormalization group sense for dimensions $D<3$, so that we expect that it generates singularities in perturbation theory 12 We conclude that the NLSM given in Eq. (3) does not contain all relevant interactions in the ordered phase below three dimensions. The reason for this is that not all approximations that are usually made in the derivation of the $\mathrm{NLSM}^{\underline{6}}$ are justified in the presence of a uniform magnetic field. In particular, it is not justified to neglect Umklapp scattering processes between transverse and longitudinal spin fluctuations involving momentum transfers across the boundary of the magnetic Brillouin zone. As an alternative to Eq. (29) it should be possible to analyse the long-wavelength staggered spin fluctuations within a phenomenological Ginzburg-Landau model with a quartic interaction term ${ }^{17}$ since this model treats the longitudinal modes as independent degrees of freedom.

The authors thank Ian Affleck for illuminating discussions. This work was supported by the DFG via Forschergruppe FOR 412.
1 N. Hasselmann and P. Kopietz, cond-mat/0511199

2 T. Holstein and H. Primakoff, Phys. Rev. 58, 1098 (1940).

3 P. W. Anderson, Phys. Rev. 86, 694 (1952).

4 S. Chakravarty, B. I. Halperin, and D. Nelson, Phys. Rev. Lett. 60, 1075 (1988); Phys. Rev. B 39, 2344 (1989).

5 I. Spremo, F. Schütz, P. Kopietz, V. Pashchenko, B. Wolf, M. Lang, J. W. Bats, C. Hu, and M. U. Schmidt, cond-mat/0505425 to appear in Phys. Rev. B.

6 S. Sachdev, Quantum Phase Transitions, (Cambridge University Press, Cambridge, 1999).

7 D. S. Fisher, Phys. Rev. B 39, 11783 (1989).

8 See, for example, J. L. McCauley, Classical Mechanics, (Cambridge University Press, Cambridge, 1997), p.212.

9 F. J. Dyson, Phys. Rev. 102, 1217 and 1230 (1956); S. V. Maleev, Zh. Eksp. Teor. Fiz. 30, 1010 (1957) [Sov. Phys. JETP 64, 654 (1958)].

10 M. E. Zhitomirsky and T. Nikuni, Phys. Rev. B 57, 5013
(1998).

11 M. E. Zhitomirsky and A. L. Chernychev, Phys. Rev. Lett. 82, 4536 (1999).

12 N. Hasselmann and P. Kopietz, in preparation.

13 F. Schütz, M. Kollar, and P. Kopietz, Phys. Rev. Lett. 91, 017205 (2003).

${ }^{14}$ We use here a basis which is rotated compared to the one used in Ref. [1]. This leads to different appearances of the minus signs in the transformation Eqs. (24|25).

15 D. I. Golosov and A. V. Chubukov, Sov. Phys. Solid State 30, 893 (1988) [Fiz. Tverd. Tela (Leningrad) 30, 1542 (1988)].

16 M. Oshikawa and I. Affleck, Phys. Rev. B 65, 134410 (2002).

17 I. Affleck, Phys. Rev. B 41, 6697 (1990); ibid. 43, 3215 (1991). 